J. Korean Math. Soc. 49 (2012), No. 2, pp. 379-394

http://dx.doi.org/10.4134/JKMS.2012.49.2.379

\title{
HOLOMORPHIC FUNCTIONS ON ALMOST COMPLEX MANIFOLDS
}

\author{
Chong-Kyu Han and Hyeseon Kim
}

\begin{abstract}
Given an almost complex structure $\left(\mathbb{C}^{m}, J\right), m \geq 2$, that is defined by setting $\theta^{\alpha}=d z^{\alpha}+a_{\beta}^{\alpha} d \bar{z}^{\beta}, \alpha=1, \ldots, m$, to be $(1,0)$-forms, we find conditions on $\left(a_{\beta}^{\alpha}\right)$ for the existence of holomorphic functions and classify the almost complex structures by type $(\nu, q)$. Then we determine types for several examples in $\mathbb{C}^{2}$ and $\mathbb{C}^{3}$ including the natural almost complex structure on $S^{6}$.
\end{abstract}

\section{Introduction}

Let $(M, J)$ be a smooth $\left(C^{\infty}\right)$ almost complex manifold of dimension $2 m$, $m \geq 2$. We are concerned in this paper with the problem of deciding the maximal number of independent holomorphic functions on $M$. Our viewpoint is purely local, thus $M$ must be regarded as a small neighborhood of a reference point and we may shrink the neighborhood finitely many times as our argument proceeds. We work in $C^{\infty}$ category; all manifolds and maps are assumed to be of differentiability class $C^{\infty}$ unless stated otherwise. There can be at most $m$ independent holomorphic functions, which is the case that the integrability condition of the Newlander-Nirenberg theorem [14] holds. A generalization due to L. Nirenberg and F. Treves (cf. [15] or [2]) states that there exist $q$ $(1 \leq q \leq m)$ independent holomorphic functions if and only if there exists a closed sub-bundle $W$ of rank $q$ of the bundle of $(1,0)$-forms, which we shall discuss in §1. In [13] O. Muškarov showed that a closed sub-bundle $W$ of $(1,0)$ forms corresponds in a natural one-to-one manner to a certain sub-bundle of the real tangent bundle $T M$ that contains the image of the Nijenhuis tensor and satisfies the Frobenius integrability condition. Given an almost complex manifold $(M, J)$ we shall construct in this paper the closed sub-bundle $W$ of differentials of the maximal set of independent holomorphic functions following

Received February 8, 2011; Revised July 30, 2011

2010 Mathematics Subject Classification. 32Q60, 32E99, 35J99.

Key words and phrases. almost complex manifolds, J-holomorphic functions, Nijenhuis tensor, Newlander-Nirenberg theorem.

First author was partially supported by NRF-Korea 2009-0070971. Second author was supported by NRF-Korea 2010-0001985. 
the method of deciding the maximal number of first integrals due to E. Car$\tan [4]$ and R. B. Gardner [8]. Instead of the Nijenhuis tensor we use the torsion tensor $d \theta \bmod \theta$, where $\theta:=\left(\theta^{1}, \ldots, \theta^{m}\right)$ are independent $(1,0)$-forms, to analyze the degree of non-integrability of $J$. We prove:

Theorem 0.1. Let $M^{2 m}, m \geq 2$, be a $C^{\infty}$ manifold with $C^{\infty}$ almost complex structure $J$. Let $\left(T^{*} M\right)^{1,0}$ be the bundle of $(1,0)$-forms. Then, under a generic assumption of non-degeneracy in each step of the construction, there exists a sequence of sub-bundles $\left(T^{*} M\right)^{1,0}:=I^{(0)} \supset I^{(1)} \supset I^{(2)} \supset \cdots$ and a nonnegative integer $\nu$ such that for $k=0,1,2, \ldots$,

i) $I^{(k+1)} \varsubsetneqq I^{(k)}$, if $k<\nu$,

ii) $I^{(k+1)}=I^{(k)}$, if $k \geq \nu$,

iii) $d I^{(k+1)} \equiv 0 \bmod I^{(k)}$.

Moreover, for each point $x \in M$, a function $u$ defined near $x$, is holomorphic if and only if $d u \in I^{(\nu)}$, thus the number of independent holomorphic functions is equal to the rank of $I^{(\nu)}$.

If $I^{(\nu)}$ has rank $q$, then we shall say $(M, J)$ has type $(\nu, q)$ (Definition 2.1). It is of type $q$ in the sense of O. Muškarov [13]. Then $W:=I^{(\nu)}$ is the desired closed sub-bundle. Locally, an almost complex structure is obtained by a small perturbation of the standard complex structure of $\mathbb{C}^{m}$ : Let $a_{j}^{k}, j, k=1, \ldots, m$, be $C^{\infty}$ complex-valued functions defined on a small neighborhood of the origin 0 of $\mathbb{C}^{m}$ such that $a_{j}^{k}(0)=0$. Let

$$
\theta^{\ell}=d z^{\ell}+\sum_{k=1}^{m} a_{k}^{\ell} d \bar{z}^{k}, \quad \ell=1, \ldots, m .
$$

Then $\theta:=\left(\theta^{1}, \ldots, \theta^{m}\right)$ uniquely determines an almost complex structure $J$ on $\mathbb{C}^{m}$ so that $\theta$ are $(1,0)$-forms. We prove:

Theorem 0.2. Suppose that $a_{k}^{j}, j, k=1, \ldots, m$, are $C^{\infty}$ complex-valued functions defined on a neighborhood of the origin 0 of $\mathbb{C}^{m}, m \geq 2$, and that $a_{k}^{j}(0)=0$. Let $J$ be the uniquely determined almost complex structure that has $(0.1)$ as $(1,0)$-forms. Suppose that $J$ has type $(\nu, q)$. Then there exist $q$ independent holomorphic functions $\zeta^{1}, \ldots, \zeta^{q}$. The condition that $J$ has type $(\nu, q)$ with $q \geq 1$ is given as a system of non-linear partial differential equations of order $\nu+1$ on $\left(a_{k}^{j}\right)$.

We use the method of prolongation of exterior differential systems as in [1], [9] and [10]: The proof of Theorem 0.2 is a complex version of the classical method of prolongation to involutive systems. In Section 3, we shall present examples of almost complex structures of various types that are obtained by small perturbations of the standard complex structures on $\mathbb{C}^{2}$ and on $\mathbb{C}^{3}$, respectively. In $\mathbb{C}^{2}$, we construct two examples of different types by computing torsion matrices. Using the classical technique of finding first integrals, we find independent holomorphic functions for each case. 


\section{Preliminaries}

Let $M$ be a smooth $\left(C^{\infty}\right)$ manifold of dimension $2 m, m \geq 2$, with smooth almost complex structure $J$. Then the complexified tangent bundle $\mathbb{C} T M$ has decomposition

$$
\mathbb{C} T M=T^{1,0}(M) \oplus T^{0,1}(M),
$$

where $T^{1,0}(M)\left(T^{0,1}(M)\right.$, respectively) is the sub-bundle of rank $m$ of eigenvectors of $J$ associated with the eigen-value $i(-i$, respectively). Dually, the complexified cotangent bundle $\mathbb{C} T^{*} M$ has decomposition:

$$
\mathbb{C} T^{*} M=\left(T^{*} M\right)^{1,0} \oplus\left(T^{*} M\right)^{0,1} .
$$

We can find real vector fields $X_{j}, j=1, \ldots, m$, so that

$$
X_{1}, J X_{1}, \ldots, X_{m}, J X_{m}
$$

span the real tangent bundle $T M$. Let $Z_{j}=\frac{1}{2}\left(X_{j}-i J X_{j}\right)$ and $\bar{Z}_{j}=\frac{1}{2}\left(X_{j}+\right.$ $\left.i J X_{j}\right)$ for each $j=1, \ldots, m$. Then $\left\{Z_{1}, \ldots, Z_{m}\right\}$ spans $T^{1,0}(M)$ and $\left\{\bar{Z}_{1}, \ldots\right.$, $\left.\bar{Z}_{m}\right\}$ spans $T^{0,1}(M)$. Let $\theta^{1}, \ldots, \theta^{m}, \bar{\theta}^{1}, \ldots, \bar{\theta}^{m}$ be the dual 1 -forms. Then the sub-bundles $\left(T^{*} M\right)^{1,0}$ and $\left(T^{*} M\right)^{0,1}$ of the complexified cotangent bundle are the linear spans of $\left\{\theta^{1}, \ldots, \theta^{m}\right\}$ and $\left\{\bar{\theta}^{1}, \ldots, \bar{\theta}^{m}\right\}$, respectively.

A complex-valued function $\zeta$ is said to be holomorphic if

$$
\bar{Z}_{j} \zeta=0, \quad j=1, \ldots, m .
$$

(1.1) is an over-determined system of linear PDEs, and thus in general, there are no solutions other than constants. Holomorphic functions $\zeta^{1}, \ldots, \zeta^{q}$ are said to be independent if

$$
d \zeta^{1} \wedge \cdots \wedge d \zeta^{q} \neq 0
$$

(1.1) is equivalent to saying that $d \zeta$ is a section of $\left(T^{*} M\right)^{1,0}$, so that there exist at most $m$ independent holomorphic functions. $J$ is said to be integrable if

$$
\left[T^{1,0}(M), T^{1,0}(M)\right] \subset T^{1,0}(M),
$$

which means that the bracket of any two sections of $T^{1,0}(M)$ is again a section of $T^{1,0}(M)$.

We consider the exterior algebra of differential forms with complex coefficients:

$$
\Omega^{*}=\Omega^{0} \oplus \Omega^{1} \oplus \cdots \oplus \Omega^{2 m},
$$

where $\Omega^{0}$ is the ring of smooth complex-valued functions and $\Omega^{r}(r=1, \ldots, 2 m)$ is the module over $\Omega^{0}$ of complex-valued smooth $r$-forms on $M$.

Definition 1.1. A subalgebra $\mathcal{I}$ of $\Omega^{*}$ is called an algebraic ideal if the following conditions hold:

i) $\mathcal{I} \wedge \Omega^{*} \subset \mathcal{I}$

ii) If $\phi=\sum_{r=0}^{2 m} \phi_{r} \in \mathcal{I}$, where $\phi_{r} \in \Omega^{r}$, then each $\phi_{r} \in \mathcal{I}$ (homogeneity condition). 
The homogeneity condition implies that $\mathcal{I}$ is two-sided, that is, $\Omega^{*} \wedge \mathcal{I} \subset \mathcal{I}$. In this paper we consider ideals generated by finitely many 1 -forms: Let $\phi=$ $\left(\phi^{1}, \ldots, \phi^{q}\right)$ be a system of elements of $\Omega^{1}$. We denote by $\mathcal{I}(\phi)$, or simply by $(\phi)$, the algebraic ideal generated by $\phi$, which is the set of all elements of $\Omega^{*}$ of the form

$$
\sum_{k=1}^{q} \phi^{k} \wedge \psi^{k}
$$

for some $\psi^{k} \in \Omega^{*}$. For two elements $\alpha$ and $\beta$ of $\Omega^{*}$

$$
\alpha \equiv \beta \bmod (\phi)
$$

means that $\alpha-\beta \in \mathcal{I}(\phi)$.

Then the integrability condition (1.2) can be written as

$$
\left[Z_{j}, Z_{k}\right] \in \Gamma\left(T^{1,0}(M)\right), \quad \forall j, k=1, \ldots, m,
$$

where $\Gamma$ denotes the set of all smooth sections. (1.3) is equivalent to

$$
d \theta^{\ell} \equiv 0 \bmod (\theta), \quad \forall \ell=1, \ldots, m,
$$

where $\theta=\left(\theta^{1}, \cdots, \theta^{m}\right)$.

Theorem 1.2 (Newlander-Nirenberg [14]). Let $\left(M^{2 m}, J\right)$ be a $C^{\infty}$ almost complex manifold. If $J$ is integrable, then there exist $m$ independent holomorphic functions.

The converse is also true, which is rather obvious. Now we fix notations: For any sub-bundle $I \subset\left(T^{*} M\right)^{1,0}$ we denote by $\underline{I}$ the module over $\Omega^{0}$ of smooth sections of $I$ and by $(I)$ the algebraic ideal of $\Omega^{*}$ generated by the smooth sections of $I$. By using Theorem 1.2 and the Frobenius theorem the following was proved in [15]:

Theorem 1.3. Suppose that $T^{\prime}$ is a sub-bundle of $\left(T^{*} M\right)^{1,0}$ of rank $q, q<m$, and that $T^{\prime}$ is closed, that is, $d \underline{T} \subset\left(T^{\prime}\right)$. Then there exist $q$ independent holomorphic functions $\zeta^{1}, \ldots, \zeta^{q}$ whose differentials $d \zeta^{1}, \ldots, d \zeta^{q}$ span $T^{\prime}$.

For any real vector fields $X$ and $Y$ the integrability condition is that

$$
[X-i J X, Y-i J Y]
$$

is a vector field of $(1,0)$-type, or equivalently,

$$
[X, Y]-[J X, J Y]=-J\{[J X, Y]+[X, J Y]\} .
$$

The Nijenhuis tensor $N$ is defined as the difference between two sides of (1.4), namely,

$$
N(X, Y):=[X, Y]+J[J X, Y]+J[X, J Y]-[J X, J Y], \quad \forall X, Y \in \Gamma(T M) .
$$

$J$ is integrable if and only if $N=0$. The following are easy to check:

i) $N(X, Y)=-N(Y, X)$, 
ii) $N(X, J Y)=N(J X, Y)=-J N(X, Y)$,

iii) If we extend $N$ complex linearly to $\mathbb{C} T M$ we have $N\left(X_{1,0}, Y_{1,0}\right)=$ $N(X, Y)_{0,1}$, where $X_{1,0}=\frac{1}{2}(X-i J X), X_{0,1}=\frac{1}{2}(X+i J X)$, and so forth.

It follows from ii) that the image of Nijenhuis tensor is $J$-invariant subset of $T M$, and therefore, at each point $x \in M$, the linear span $\mathcal{L} N(x)$ of the images of the Nijenhuis tensor is even dimensional. $\mathcal{L} N(x)$ is the obstruction to the existence of holomorphic functions (cf. [11]).

Definition 1.4. $\operatorname{rank} N$ is the map: $x \mapsto \operatorname{dim}_{\mathbb{R}} \mathcal{L} N(x)$.

This map is lower semi-continuous (cf. [12]). If rank $N$ is constant, then $\mathcal{L} N$ is a sub-bundle of $T M$. Based on Theorem 1.3 Muškarov proved the following.

Theorem $1.5([13])$. Let $\left(M^{2 m}, J\right)$ be a $C^{\infty}$ almost complex manifold with Nijenhuis tensor $N$. Suppose that $\operatorname{rank} N=2(m-q)$ is constant. Then the number of independent holomorphic functions is at most q. There exist $q$ independent holomorphic functions $\zeta^{1}, \ldots, \zeta^{q}$ if and only if the sub-bundle $\mathcal{L} N \subset T M$ has the following properties:

i) $\mathcal{L} N$ is involutive,

ii) $[X, Y]+J[X, J Y] \in \Gamma(\mathcal{L} N)$ for all $X \in \Gamma(\mathcal{L} N)$ and all $Y \in \Gamma(T M)$.

\section{Nullity of torsion tensor and type of almost complex structures}

We study the existence of holomorphic functions in the framework of CartanGardner theory [4] and [8] on first integrals (cf. [3]). We also make use of Theorem 1.3. First of all we prove Theorem 0.1.

Proof of Theorem 0.1. We shall find the largest closed sub-bundle of $\left(T^{*} M\right)^{1,0}$ starting with $I=I^{(0)}=\left(T^{*} M\right)^{1,0}$ : The exterior derivative $d: \underline{I} \rightarrow \Omega^{2}$ is not a module homomorphism, but composition with the projection

$$
\underline{\mathrm{I}} \stackrel{d}{\rightarrow} \Omega^{2} \stackrel{\pi}{\rightarrow} \Omega^{2} /(I)
$$

is a $\Omega^{0}$-module homomorphism. Let $\delta=\pi \circ d$. Consider the submodule $\underline{\mathrm{I}}^{(1)}:=$ ker $\delta$ of $\mathrm{I}$. We assume that $\mathrm{I}^{(1)}$ has constant rank on $M$, hence defines a sub-bundle $I^{(1)}$ of $\left(T^{*} M\right)^{1,0}$. We have a short exact sequence of $\Omega^{0}$-modules

$$
0 \rightarrow \underline{\mathrm{I}}^{(1)} \rightarrow \underline{\mathrm{I}} \stackrel{\delta}{\rightarrow} d \underline{\mathrm{I}} /(I) \rightarrow 0 .
$$

The sub-bundle $I^{(1)}$ is called the first derived system of $\left(T^{*} M\right)^{1,0}$. Assuming that $\underline{I}^{(k-1)}$ has constant rank, we define inductively the $k$-th derived system $I^{(k)}$ by

$$
0 \rightarrow \underline{\mathrm{I}}^{(k)} \rightarrow \underline{\mathrm{I}}^{(k-1)} \stackrel{\delta}{\rightarrow} d \underline{\mathrm{I}}^{(k-1)} /\left(I^{(k-1)}\right) \rightarrow 0 .
$$

Let $\nu$ be the smallest integer with $I^{(\nu)}=I^{(\nu+1)}$. Then we have a sequence of sub-bundles

$$
\left(T^{*} M\right)^{1,0}:=I:=I^{(0)} \supset I^{(1)} \supset \cdots \supset I^{(\nu-1)} \supset I^{(\nu)} .
$$


Notice that $d \underline{\mathrm{I}}^{(\nu)} \subset\left(I^{(\nu)}\right)$, that is, $I^{(\nu)}$ is closed. Assume that $\underline{\mathrm{I}}^{(\nu)}$ has constant rank $q$. Then by Theorem 1.3 there exist independent holomorphic functions $\zeta^{1}, \ldots, \zeta^{q}$ such that $d \zeta^{1}, \ldots, d \zeta^{q}$ generates $I^{(\nu)}$. For a complex-valued function $f$ defined on $M$ we recall that

$$
\begin{aligned}
f \text { is holomorphic } & \Longleftrightarrow d f \in I^{(0)}, \text { by definition } \\
& \Longleftrightarrow d f \in I^{(k)}, \forall k=1,2, \ldots, \text { since } d f \text { is a closed } 1 \text {-form } \\
& \Longleftrightarrow d f \in I^{(\nu)} .
\end{aligned}
$$

Hence, the number of independent holomorphic functions is same as the rank of $I^{(\nu)}$, which completes the proof of Theorem 0.1 .

Definition 2.1. $(M, J)$ is said to be of type $(\nu, q)$ if $I^{(\nu)}$ has rank $q$.

Given $(M, J)$ we now construct the sequence (2.1) of sub-bundles and decide the type $(\nu, q)$. Let $\theta=\left(\theta^{1}, \ldots, \theta^{m}\right)$ be independent $(1,0)$-forms. We set

$$
d \theta^{\ell} \equiv \sum_{j<k} T_{j k}^{\ell} \bar{\theta}^{j} \wedge \bar{\theta}^{k} \bmod (\theta),
$$

where $\ell, j, k=1, \ldots, m$. In matrices $(2.2)$ can be written as

$$
\left[\begin{array}{c}
d \theta^{1} \\
\vdots \\
d \theta^{m}
\end{array}\right] \equiv \underbrace{\left[\begin{array}{cccc}
T_{12}^{1} & T_{13}^{1} & \cdots & T_{m-1, m}^{1} \\
\vdots & \vdots & \ddots & \vdots \\
T_{12}^{m} & T_{13}^{m} & \cdots & T_{m-1, m}^{m}
\end{array}\right]}_{\mathcal{T}}\left[\begin{array}{c}
\bar{\theta}^{1} \wedge \bar{\theta}^{2} \\
\bar{\theta}^{1} \wedge \bar{\theta}^{3} \\
\vdots \\
\bar{\theta}^{m-1} \wedge \bar{\theta}^{m}
\end{array}\right] \bmod (\theta) .
$$

The $m \times\left(\begin{array}{c}m \\ 2\end{array}\right)$ matrix $\mathcal{T}$ is called the torsion of $J$ with respect to the coframe $\theta$. Like the Nijenhuis tensor the torsion also measures the non-integrability of $J$ : If $\mathcal{T}$ has rank zero, that is, all the entries of $\mathcal{T}$ are zeros, then $J$ is integrable. Relation between $\mathcal{T}$ and $N$ is

$$
N\left(\bar{Z}_{j}, \bar{Z}_{k}\right)=-4 \sum_{\ell=1}^{m} T_{j k}^{\ell} Z_{\ell},
$$

which is easy to check.

Proposition 2.2. If there exist independent holomorphic functions $\zeta^{1}, \ldots, \zeta^{q}$, then $\operatorname{rank} \mathcal{T} \leq m-q$.

Proof. For each $\lambda=1, \ldots, q$, that $\zeta^{\lambda}$ is holomorphic implies

$$
d \zeta^{\lambda}=\sum_{\ell=1}^{m} b_{\ell}^{\lambda} \theta^{\ell}
$$


for some functions $b_{\ell}^{\lambda}$. Now by applying $d$ to (2.4) and by substituting (2.3) for $d \theta^{\ell}$ we have

$$
\begin{aligned}
0 & \equiv \sum_{\ell=1}^{m} b_{\ell}^{\lambda} d \theta^{\ell} \bmod (\theta) \\
& \equiv \sum_{\ell=1}^{m} \sum_{j<k} b_{\ell}^{\lambda} T_{j k}^{\ell} \bar{\theta}^{j} \wedge \bar{\theta}^{k} \bmod (\theta),
\end{aligned}
$$

so that

$$
\left[\begin{array}{c}
0 \\
\vdots \\
0
\end{array}\right] \equiv \underbrace{\left[\begin{array}{ccc}
b_{1}^{1} & \cdots & b_{m}^{1} \\
\vdots & & \vdots \\
b_{1}^{q} & \cdots & b_{m}^{q}
\end{array}\right]}_{\mathcal{B}} \underbrace{\left[\begin{array}{cccc}
T_{12}^{1} & T_{13}^{1} & \cdots & T_{m-1, m}^{1} \\
\vdots & \vdots & \ddots & \vdots \\
T_{12}^{m} & T_{13}^{m} & \cdots & T_{m-1, m}^{m}
\end{array}\right]}_{\mathcal{T}}\left[\begin{array}{c}
\bar{\theta}^{1} \wedge \bar{\theta}^{2} \\
\bar{\theta}^{1} \wedge \bar{\theta}^{3} \\
\vdots \\
\bar{\theta}^{m-1} \wedge \bar{\theta}^{m}
\end{array}\right] \bmod (\theta) .
$$

Since the 2-forms $\bar{\theta}^{j} \wedge \bar{\theta}^{k}, j<k$, are independent, we have

$$
\mathcal{B T}=0 .
$$

Since $d \zeta^{1} \wedge \cdots \wedge d \zeta^{q} \neq 0$ the rows of $\mathcal{B}$ are independent and each row of $\mathcal{B}$ is a null vector of $\mathcal{T}$. Therefore, dimension of the null space of $\mathcal{T}$ is at least $q$, which implies that $\operatorname{rank} \mathcal{T} \leq m-q$.

Now assume $\operatorname{rank} \mathcal{T}=r, r<m$, is constant. Then by Proposition 2.2 there exist at most $m-r$ independent holomorphic functions.

Proposition 2.3. Construction of $I^{(1)}$ : Suppose the dimension of the null space of the matrix $\mathcal{T}$ is $q_{1}$ and that the row vectors $\vec{b}^{\lambda}=\left(b_{1}^{\lambda}, \ldots, b_{m}^{\lambda}\right), \lambda=$ $1, \ldots, q_{1}$, are independent null vectors of $\mathcal{T}$ as the rows of $\mathcal{B}$ in (2.5). Let

$$
\phi^{\lambda}=\sum_{\ell=1}^{m} b_{\ell}^{\lambda} \theta^{\ell}, \quad \lambda=1, \ldots, q_{1} .
$$

Then $I^{(1)}$ is spanned by $\left\{\phi^{\lambda}\right\}$.

Proof. For each $\lambda=1, \ldots, q_{1}$

$$
\begin{aligned}
d \phi^{\lambda} & \equiv \sum_{\ell=1}^{m} b_{\ell}^{\lambda} d \theta^{\ell} \bmod (\theta) \\
& \equiv \sum_{\ell=1}^{m} \sum_{\substack{j<k \\
j, k=1, \ldots, m}} b_{\ell}^{\lambda} T_{j k}^{\ell} \bar{\theta}^{j} \wedge \bar{\theta}^{k} \bmod (\theta) \\
& \equiv 0 \bmod (\theta),
\end{aligned}
$$

and therefore, $\phi^{\lambda} \in \underline{I}^{(1)}$. Conversely, if $\phi:=\sum_{\ell=1}^{m} b_{\ell} \theta^{\ell}$ is in $\underline{I}^{(1)}$, then by the same calculation as in (2.6) the row vector $\left(b_{1}, \ldots, b_{m}\right)$ is a null vector of $T$. 
Inductively, we have:

Proposition 2.4. Construction of $I^{(k+1)}$ from $I^{(k)}$ : Suppose $I^{(k)}$ has rank $q_{k}$. Let $\psi:=\left(\psi^{1}, \ldots, \psi^{q_{k}}\right)$ be a set of generators of $\underline{I}^{(k)}$. Complete $\psi$ to a coframe $\left(\psi^{1}, \ldots, \psi^{q_{k}}, \omega^{1}, \ldots, \omega^{r}\right)$, where $r=2 m-q_{k}$. We find the torsion $\tau$ for the Pfaffian system $\psi$ by setting

$$
\left[\begin{array}{c}
d \psi^{1} \\
\vdots \\
d \psi^{q_{k}}
\end{array}\right] \equiv \underbrace{\left[\begin{array}{cccc}
\tau_{12}^{1} & \tau_{13}^{1} & \cdots & \tau_{r-1, r}^{1} \\
\vdots & \vdots & \ddots & \vdots \\
\tau_{12}^{q_{k}} & \tau_{13}^{q_{k}} & \cdots & \tau_{r-1, r}^{q_{k}}
\end{array}\right]}_{\tau}\left[\begin{array}{c}
\omega^{1} \wedge \omega^{2} \\
\omega^{1} \wedge \omega^{3} \\
\vdots \\
\omega^{r-1} \wedge \omega^{r}
\end{array}\right] \bmod (\psi) .
$$

Here, the torsion matrix $\tau$ has size $q_{k} \times\left(\begin{array}{l}r \\ 2\end{array}\right)$. Let $q_{k+1}$ be the nullity of the matrix $\tau$ and let

$$
\vec{c}^{\mu}=\left(c_{1}^{\mu}, \ldots, c_{q_{k}}^{\mu}\right), \quad \mu=1, \ldots, q_{k+1}
$$

be the null vectors of $\tau$, namely, $c_{\lambda}^{\mu}$ satisfies

$$
\sum_{\lambda=1}^{q_{k}} c_{\lambda}^{\mu} \tau_{i j}^{\lambda}=0
$$

for each pair $i, j=1, \ldots, r$ with $i<j$. Then $q_{k+1} 1$-forms

$$
\sum_{\lambda=1}^{q_{k}} c_{\lambda}^{\mu} \psi^{\lambda}, \quad \mu=1, \ldots, q_{k+1},
$$

$\operatorname{span} I^{(k+1)}$

Locally, an almost complex structure is obtained by a small perturbation of the standard complex structure of $\mathbb{C}^{m}$ : Let $a_{j}^{k}, j, k=1, \ldots, m$, be $C^{\infty}$ complex-valued functions defined on a small neighborhood of the origin 0 of $\mathbb{C}^{m}$ such that $a_{j}^{k}(0)=0$. Consider the system of linear PDEs

$$
\bar{Z}_{j} \zeta=0, \quad j=1, \ldots, m,
$$

where

$$
\bar{Z}_{j}=\frac{\partial}{\partial \bar{z}^{j}}-\sum_{k=1}^{m} a_{j}^{k} \frac{\partial}{\partial z^{k}}
$$

are the perturbed Cauchy-Riemann operators. Then solving (2.7) is equivalent to finding a complex-valued function $\zeta$ such that $d \zeta \in \mathcal{I}\left(\theta^{1}, \ldots, \theta^{m}\right)$, where

$$
\theta^{\ell}=d z^{\ell}+\sum_{k=1}^{m} a_{k}^{\ell} d \bar{z}^{k} .
$$

Now let $M=\mathbb{C}^{m}$ and let $J$ be the uniquely determined almost complex structure whose $(1,0)$-forms are $(2.8)$. Let $(\nu, q)$ be the type of the almost complex structure determined by perturbation $\left(a_{k}^{j}\right)$. If $\nu=0$, then $d \underline{\mathrm{I}} \subset(I)$, which is 
the integrability condition of the Newlander-Nirenberg theorem. We see that $\nu=0$ if and only if

$$
d \theta^{\ell} \equiv 0 \bmod (\theta)
$$

$(2.9)$ is a system of non-linear partial differential equations of first order on $\left(a_{k}^{j}\right)$. If $(M, J)$ has type $(\nu, q)$, the system of functions $\left(a_{k}^{j}\right)$ undergoes a process of algebraic operation and differentiation $\nu+1$ times. In particular, $\delta=\pi \circ d$ is applied $\nu+1$ times to $\theta$ 's. Thus we proved Theorem 0.2 .

\section{Cases $\left(\mathbb{C}^{2}, J\right)$ and $\left(\mathbb{C}^{3}, J\right)$ and examples}

Let $a:=\left(a_{k}^{j}\right)$ be as in (2.8). Solving (2.8) and its complex conjugate for $d z^{j}$ and $d \bar{z}^{j}$ we have

$$
d z^{j}=\sum_{k=1}^{m} c_{k}^{j} \theta^{k}+\sum_{k=1}^{m} \epsilon_{k}^{j} \bar{\theta}^{k},
$$

where $c_{k}^{j}$ and $\epsilon_{k}^{j}$ are rational functions in $a_{k}^{j}$ and their complex conjugates such that

$$
\begin{aligned}
& c_{k}^{j}(0)=\delta_{k}^{j} \quad(\text { Kronecker delta }), \\
& \epsilon_{k}^{j}(0)=0 .
\end{aligned}
$$

Applying $d$ to (2.8) we have

$$
\begin{aligned}
d \theta^{\ell} & =\sum_{k=1}^{m} d a_{k}^{\ell} \wedge d \bar{z}^{k} \\
& =\sum_{k=1}^{m} \sum_{j=1}^{m}\left(\frac{\partial a_{k}^{\ell}}{\partial z^{j}} d z^{j}+\frac{\partial a_{k}^{\ell}}{\partial \bar{z}^{j}} d \bar{z}^{j}\right) \wedge d \bar{z}^{k}
\end{aligned}
$$

substitute (3.1) and its complex-conjugate for $d z^{j}$ and $d \bar{z}^{j}$, respectively,

$$
\equiv \sum_{\substack{j<k \\ j, k=1, \ldots, m}} T_{j k}^{\ell} \bar{\theta}^{j} \wedge \bar{\theta}^{k} \bmod (\theta),
$$

where $T_{j k}^{\ell}$ are linear in the first order partial derivatives of $\left(a_{k}^{j}\right)$ with coefficients that are rational in $\left(a_{k}^{j}\right)$ and

$$
T_{j k}^{\ell}(0)=\left(\frac{\partial a_{k}^{\ell}}{\partial \bar{z}^{j}}-\frac{\partial a_{j}^{\ell}}{\partial \bar{z}^{k}}\right)(0) .
$$

\subsection{Case $m=2$}

(2.3) with $m=2$ is

$$
\left[\begin{array}{l}
d \theta^{1} \\
d \theta^{2}
\end{array}\right] \equiv\left[\begin{array}{l}
T^{1} \\
T^{2}
\end{array}\right] \bar{\theta}^{1} \wedge \bar{\theta}^{2} \quad \bmod (\theta) .
$$


$J$ is integrable if and only if both $T^{1}$ and $T^{2}$ are identically zero. If $\mathcal{T}:=\left[\begin{array}{l}T^{1} \\ T^{2}\end{array}\right]$ has constant rank 1 , let $b:=\frac{T^{2}}{T^{1}}$ assuming $T^{1} \neq 0$. Then $I^{(1)}$ is generated by

$$
\phi:=b \theta^{1}-\theta^{2} .
$$

$I^{(1)}$ is integrable if and only if $d \phi \equiv 0 \bmod (\phi)$, that is,

$$
d \phi \wedge \phi=0 .
$$

Notice that (3.2) involves first order derivatives of $T^{j}, j=1,2$, and therefore, a system of second order partial differential equations on $\left(a_{k}^{j}\right)$. If $\left(a_{k}^{j}\right)$ satisfy (3.2), then $\nu=1$ and $q=1$. In this case, there exists one independent holomorphic function. If $\left(a_{k}^{j}\right)$ do not satisfy (3.2), then there is no holomorphic function other than constants. Assuming that $I^{(1)}$ has constant rank, there are four possibilities as in the following table.

$$
\operatorname{dim} I \operatorname{dim} I^{(1)} \quad \operatorname{dim} I^{(2)} \quad \text { type } \begin{gathered}
\text { number of } \\
\text { holomorphic functions }
\end{gathered} \quad \operatorname{PDE} \text { for }\left(a_{k}^{j}\right)
$$

$\begin{array}{ccccccc}\text { case } 1 & 2 & 2 & & (0,2) & 2 & \text { 1st order } \\ \text { case } 2 & 2 & 1 & 1 & (1,1) & 1 & \text { 2nd order } \\ \text { case } 3 & 2 & 1 & 0 & (2,0) & 0 & \\ \text { case } 4 & 2 & 0 & 0 & (1,0) & 0\end{array}$

Example 3.1. Consider $\left(\mathbb{C}^{2}, J\right)$ where $J$ is defined by declaring

$$
\begin{aligned}
& \theta^{1}=d z^{1}+z^{1} d \bar{z}^{2}, \\
& \theta^{2}=d z^{2}
\end{aligned}
$$

to be $(1,0)$-forms.

By computing $d \theta \bmod (\theta)$, we see that $T^{1}=T^{2}=0$, so that $J$ has type $(0,2)$. Hence, there exist two independent holomorphic functions. Obviously, $z^{2}$ is a holomorphic function. Using the classical technique of finding first integrals as in [5] the other one can be found as follows: The $(0,1)$-vector fields are

$$
\begin{aligned}
& \bar{Z}_{1}=\frac{\partial}{\partial \bar{z}^{1}}, \\
& \bar{Z}_{2}=\frac{\partial}{\partial \bar{z}^{2}}-z^{1} \frac{\partial}{\partial z^{1}} .
\end{aligned}
$$

A first integral of $\bar{Z}_{2}$ is obtained by solving

$$
\frac{d \bar{z}^{2}}{1}=\frac{d z^{1}}{-z^{1}}
$$

whose solution is $\bar{z}^{2}+\log z^{1}=$ constant. To avoid singularity at the origin we exponentiate $\bar{z}^{2}+\log z^{1}$, to obtain

$$
\zeta\left(z^{1}, \bar{z}^{1}, z^{2}, \bar{z}^{2}\right)=z^{1} e^{\bar{z}^{2}}
$$

as another holomorphic function. 
Example 3.2. Consider $\left(\mathbb{C}^{2}, J\right)$ with $(1,0)$-forms

$$
\begin{aligned}
& \theta^{1}=d z^{1}+z^{1} d \bar{z}^{2}, \\
& \theta^{2}=d z^{2}+\bar{z}^{2} d \bar{z}^{1} .
\end{aligned}
$$

Then we have

$$
\left[\begin{array}{l}
d \theta^{1} \\
d \theta^{2}
\end{array}\right] \equiv\left[\begin{array}{c}
0 \\
-\left|1-z^{1} z^{2}\right|^{-2}
\end{array}\right] \bar{\theta}^{1} \wedge \bar{\theta}^{2} \bmod (\theta) .
$$

Then the first derived system $I^{(1)}$ is generated by $\theta^{1}$ and the number of independent holomorphic functions is at most one. Since

$$
d \theta^{1} \equiv 0 \bmod \left(\theta^{1}\right),
$$

$J$ has type $(1,1)$. As in Example $3.1 \zeta\left(z^{1}, \bar{z}^{1}, z^{2}, \bar{z}^{2}\right)=z^{1} e^{\bar{z}^{2}}$ is a holomorphic function.

\subsection{Case $m=3$}

(2.3) with $m=3$ is

$$
\left[\begin{array}{c}
d \theta^{1} \\
d \theta^{2} \\
d \theta^{3}
\end{array}\right] \equiv \underbrace{\left[\begin{array}{ccc}
T_{12}^{1} & T_{13}^{1} & T_{23}^{1} \\
T_{12}^{2} & T_{13}^{2} & T_{23}^{2} \\
T_{12}^{3} & T_{13}^{3} & T_{23}^{3}
\end{array}\right]}_{\mathcal{T}}\left[\begin{array}{l}
\bar{\theta}^{1} \wedge \bar{\theta}^{2} \\
\bar{\theta}^{1} \wedge \bar{\theta}^{3} \\
\bar{\theta}^{2} \wedge \bar{\theta}^{3}
\end{array}\right] \quad \bmod (\theta) .
$$

$J$ is integrable if and only if $\mathcal{T}$ is identically zero. Let $\mathcal{T}^{j}$ be the $j$-th row of $\mathcal{T}$ for $j=1,2,3$. Assuming rank $\mathcal{T}$ is constant we shall find generators of $I^{(1)}$. If rank $\mathcal{T}=3$, there is no holomorphic function other than constants by Proposition 2.2. If rank $\mathcal{T}=2$, we may assume that $\mathcal{T}^{3}=b_{1} \mathcal{T}^{1}+b_{2} \mathcal{T}^{2}$ for some complex-valued functions $b_{1}$ and $b_{2}$. Then

$$
\phi:=b_{1} \theta^{1}+b_{2} \theta^{2}-\theta^{3}
$$

generates $I^{(1)} . I^{(1)}$ is integrable if and only if $d \phi \wedge \phi=0$, which is the case that $\nu=1$ and $q=1$. If $\operatorname{rank} \mathcal{T}=1$, then there exist at most 2 independent holomorphic functions by Proposition 2.2. Assuming the third row of $\mathcal{T}$ is non-zero, we let

$$
\mathcal{T}^{1}=b_{1} \mathcal{T}^{3}, \quad \mathcal{T}^{2}=b_{2} \mathcal{T}^{3} .
$$

Then $I^{(1)}$ is generated by two 1 -forms

$$
\begin{aligned}
\phi^{1} & :=\theta^{1}-b_{1} \theta^{3}, \\
\phi^{2} & :=\theta^{2}-b_{2} \theta^{3} .
\end{aligned}
$$

$I^{(1)}$ is integrable if and only if

$$
\left\{\begin{array}{l}
d \phi^{1} \wedge \phi^{1} \wedge \phi^{2}=0 \\
d \phi^{2} \wedge \phi^{1} \wedge \phi^{2}=0
\end{array}\right.
$$

This is the case that $\nu=1$ and $q=2$. 
Assuming $I^{(k)}, k=1,2,3$, are of constant dimensions there are eight possible cases as the following table shows.

$\operatorname{dim} I \operatorname{dim} I^{(1)} \quad \operatorname{dim} I^{(2)} \quad \operatorname{dim} I^{(3)} \quad$ type $\begin{gathered}\text { number of } \\ \text { holomorphic functions }\end{gathered} \quad$ PDE for $\left(a_{k}^{j}\right)$

\begin{tabular}{|c|c|c|c|c|c|c|c|}
\hline case 1 & 3 & 3 & & & $(0,3)$ & 3 & 1st order \\
\hline case 2 & 3 & 2 & 2 & & $(1,2)$ & 2 & 2nd order \\
\hline case 3 & 3 & 2 & 1 & 1 & $(2,1)$ & 1 & 3rd order \\
\hline case 4 & 3 & 2 & 1 & 0 & $(3,0)$ & 0 & \\
\hline case 5 & 3 & 2 & 0 & & $(2,0)$ & 0 & \\
\hline case 6 & 3 & 1 & 1 & & $(1,1)$ & 1 & 2nd order \\
\hline case 7 & 3 & 1 & 0 & & $(2,0)$ & 0 & \\
\hline case 8 & 3 & 0 & & & $(1,0)$ & 0 & \\
\hline
\end{tabular}

\section{Non-integrability of $S^{6}$}

Let $S^{6}$ be the unit sphere in the real Euclidean space $E(7)=\left\{\left(x^{1}, \ldots, x^{7}\right)\right\}$. In this section we discuss the natural almost complex structure on $S^{6}$ that has been studied in [7] and [6]. This almost complex structure is defined by the Cayley product of $E(7)$ and turned out to be non-integrable as shown in [13]. We present another proof of the non-integrability: we shall prove that even locally there are no holomorphic functions other than constants by using Theorem 0.1 and Theorem 0.2. In $E(7)$ consider an ortho-normal frame

$$
e_{j}=\sum_{\lambda=1}^{7} a_{j}^{\lambda} \frac{\partial}{\partial x^{\lambda}}, \quad j=1, \ldots, 7 .
$$

Then the dual 1-forms are

$$
\phi^{k}=\sum_{\mu=1}^{7} a_{k}^{\mu} d x^{\mu}, \quad k=1, \ldots, 7 .
$$

Since $e_{1}, \ldots, e_{7}$ may be considered as pure imaginary Cayley numbers, the vector cross product is naturally defined by

$$
V \times W=\sum_{j, k=1, j \neq k}^{7} v^{j} w^{k} e_{j} \cdot e_{k},
$$

where $V=v^{1} e_{1}+\cdots+v^{7} e_{7}, W=w^{1} e_{1}+\cdots+w^{7} e_{7}$, and $\cdot$ is the Cayley product (cf. [6] or [7]). At a point $x \in S^{6}$, the tangent space $T_{x} S^{6}$ is identified with the subspace $\langle x\rangle^{\perp}$ of $E(7)$. Assume $e_{1}, \ldots, e_{6}$ are tangent to $S^{6}$ at $x$. Then an almost complex structure $J$ at $x \in S^{6}$ is defined by $J_{x} X=x \times X$ for $X \in \Gamma\left(T_{x} S^{6}\right)$. For $x=\left(x^{1}, \ldots, x^{7}\right)$ in a neighborhood of $(0, \ldots, 0,1)$ we express $J_{x}$ as a matrix with respect to the basis $e_{1}, \ldots, e_{6}$ so that $J_{x} e_{j}$ is the 
$j$-th column of the following matrix:

$(4.3)$

$$
\left(J_{\mu}^{\nu}\right)(x):=\left[\begin{array}{cccccc}
-\frac{x^{1} x^{6}}{x^{7}} & -x^{3}-\frac{x^{2} x^{6}}{x^{7}} & x^{2}-\frac{x^{3} x^{6}}{x^{7}} & -x^{5}-\frac{x^{4} x^{6}}{x^{7}} & x^{4}-\frac{x^{5} x^{6}}{x^{7}} & -x^{7}-\frac{\left(x^{6}\right)^{2}}{x^{7}} \\
x^{3}-\frac{x^{1} x^{5}}{x^{7}} & -\frac{x^{2} x^{5}}{x^{7}} & -x^{1}-\frac{x^{3} x^{5}}{x^{7}} & x^{6}-\frac{x^{4} x^{5}}{x^{7}} & -x^{7}-\frac{\left(x^{5}\right)^{2}}{x^{7}} & -x^{4}-\frac{x^{5} x^{6}}{x^{7}} \\
-x^{2}-\frac{x^{1} x^{4}}{x^{7}} & x^{1}-\frac{x^{2} x^{4}}{x^{7}} & -\frac{x^{3} x^{4}}{x^{7}} & -x^{7}-\frac{\left.x^{4}\right)^{2}}{x^{7}} & -x^{6}-\frac{x^{4} x^{5}}{x^{7}} & x^{5}-\frac{x^{4} x^{6}}{x^{7}} \\
x^{5}+\frac{x^{1} x^{3}}{x^{7}} & -x^{6}+\frac{x^{2} x^{3}}{x^{7}} & x^{7}+\frac{\left(x^{3}\right)^{2}}{x^{7}} & \frac{x^{3} x^{4}}{x^{7}} & -x^{1}+\frac{x^{3} x^{5}}{x^{7}} & x^{2}+\frac{x^{3} x^{6}}{x^{7}} \\
-x^{4}+\frac{x^{1} x^{2}}{x^{7}} & x^{7}+\frac{\left(x^{2}\right)^{2}}{x^{7}} & x^{6}+\frac{x^{2} x^{3}}{x^{7}} & x^{1}+\frac{x^{2} x^{4}}{x^{7}} & \frac{x^{2} x^{5}}{x^{7}} & -x^{3}+\frac{x^{2} x^{6}}{x^{7}} \\
x^{7}+\frac{\left(x^{2}\right)^{2}}{x^{7}} & x^{4}+\frac{x^{1} x^{2}}{x^{7}} & -x^{5}+\frac{x^{1} x^{3}}{x^{7}} & -x^{2}+\frac{x^{1} x^{4}}{x^{7}} & x^{3}+\frac{x^{1} x^{5}}{x^{7}} & \frac{x^{1} x^{6}}{x^{7}}
\end{array}\right] .
$$

We note that

$$
\left(J_{\mu}^{\nu}\right)(0, \ldots, 0,1)=\left[\begin{array}{cccccc}
0 & 0 & 0 & 0 & 0 & -1 \\
0 & 0 & 0 & 0 & -1 & 0 \\
0 & 0 & 0 & -1 & 0 & 0 \\
0 & 0 & 1 & 0 & 0 & 0 \\
0 & 1 & 0 & 0 & 0 & 0 \\
1 & 0 & 0 & 0 & 0 & 0
\end{array}\right]
$$

so that $J e_{1}=e_{6}, J e_{2}=e_{5}, J e_{3}=e_{4}$ at the reference point. Therefore, $(0,1)$-vector fields of this $J$ are generated by

$$
\bar{Z}_{1}=e_{1}+i J e_{1}, \bar{Z}_{2}=e_{2}+i J e_{2} \text {, and } \bar{Z}_{3}=e_{3}+i J e_{3} .
$$

Let $J^{*}: T^{*} M \rightarrow T^{*} M$ be the dual transformation of $J$. Then

$$
\theta^{1}=\phi^{1}-i J^{*} \phi^{1}, \theta^{2}=\phi^{2}-i J^{*} \phi^{2} \text {, and } \theta^{3}=\phi^{3}-i J^{*} \phi^{3}
$$

annihilate $\bar{Z}_{j}, j=1,2,3$. Therefore, $\theta=\left(\theta^{1}, \theta^{2}, \theta^{3}\right)$ are independent $(1,0)$ forms. Now we shall show that the torsion, $d \theta \bmod \theta$, has full rank, which implies there are no holomorphic functions other than constants. Since $J e_{\mu}=$ $\sum_{\nu=1}^{6} J_{\mu}^{\nu} e_{\nu}$ we have

$$
J^{*} \phi^{\eta}=\sum_{\lambda=1}^{6} J_{\lambda}^{\eta} \phi^{\lambda} .
$$

By applying $d$ to (4.4) after substituting (4.5) for $J^{*} \phi^{\eta}$ we have

$$
d \theta^{\eta}=d \phi^{\eta}-i \sum_{\lambda=1}^{6} d J_{\lambda}^{\eta} \wedge \phi^{\lambda}-i \sum_{\lambda=1}^{6} J_{\lambda}^{\eta} d \phi^{\lambda} .
$$

To compute $d \phi^{\eta}$ 's of the right hand side of (4.6) write (4.2) in matrices as

$$
\underbrace{\left[\begin{array}{c}
\phi^{1} \\
\vdots \\
\phi^{7}
\end{array}\right]}_{\phi}=\underbrace{\left[\begin{array}{ccc}
a_{1}^{1} & \cdots & a_{1}^{7} \\
\vdots & \ddots & \vdots \\
a_{7}^{1} & \cdots & a_{7}^{7}
\end{array}\right]}_{\mathcal{A}} \underbrace{\left[\begin{array}{c}
d x^{1} \\
\vdots \\
d x^{7}
\end{array}\right]}_{d \mathcal{X}}
$$


and then apply $d$ to (4.7), to obtain

$$
\begin{aligned}
d \phi & =d \mathcal{A} \wedge d \mathcal{X} \\
& =d \mathcal{A} \mathcal{A}^{-1} \phi \\
& =d \mathcal{A} \mathcal{A}^{T} \phi, \quad \text { since } \mathcal{A} \in O(7) .
\end{aligned}
$$

Now coming back to (4.1), we make a special choice of frame $\left(e_{1}, \ldots, e_{7}\right)$ as follows:

(i) $e_{7}$ is normal and $e_{1}, \ldots, e_{6}$ are tangent to $S^{6}$.

(ii) For each $k=1, \ldots, 6$, let $A^{k}:=\left(\sum_{i=k}^{7}\left(x^{i}\right)^{2}\right)^{1 / 2}$. Then $e_{7}=\frac{1}{A^{1}}\left(x^{1}, \ldots\right.$, $\left.x^{7}\right)$. Let $e_{6}=\left(0, \ldots, 0, \frac{x^{7}}{A^{6}},-\frac{x^{6}}{A^{6}}\right)$. Then $e_{6}$ is a unit vector that is perpendicular to $e_{7}$.

(iii) For each $k=1, \ldots, 5$, let $e_{k}=(\underbrace{0, \ldots, 0}_{k-1}, \frac{A^{k+1}}{A^{k}},-\frac{x^{k} x^{k+1}}{A^{k} A^{k+1}}, \ldots,-\frac{x^{k} x^{7}}{A^{k} A^{k+1}})$.

Then $e_{k}$ is a unit vector that is perpendicular to $e_{k+1}, \ldots, e_{7}$.

Thus we have

$$
\mathcal{A}=\left[\begin{array}{ccccccc}
\frac{A^{2}}{A^{1}} & -\frac{x^{1} x^{2}}{A^{1} A^{2}} & -\frac{x^{1} x^{3}}{A^{1} A^{2}} & -\frac{x^{1} x^{4}}{A^{1} A^{2}} & -\frac{x^{1} x^{5}}{A^{1} A^{2}} & -\frac{x^{1} x^{6}}{A^{1} A^{2}} & -\frac{x^{1} x^{7}}{A^{1} A^{2}} \\
0 & \frac{A^{3}}{A^{2}} & -\frac{x^{2} x^{3}}{A^{2} A^{3}} & -\frac{x^{2} x^{4}}{A^{2} A^{3}} & -\frac{x^{2} x^{5}}{A^{2} A^{3}} & -\frac{x^{2} x^{6}}{A^{2} A^{3}} & -\frac{x^{2} x^{7}}{A^{2} A^{3}} \\
0 & 0 & \frac{A^{4}}{A^{3}} & -\frac{x^{3} x^{4}}{A^{3} A^{4}} & -\frac{x^{3} x^{5}}{A^{3} A^{4}} & -\frac{x^{3} x^{6}}{A^{3} A^{4}} & -\frac{x^{3} x^{7}}{A^{3} A^{4}} \\
0 & 0 & 0 & \frac{A^{5}}{A^{4}} & -\frac{x^{4} x^{5}}{A^{4} A^{5}} & -\frac{x^{4} x^{6}}{A^{4} A^{5}} & -\frac{x^{4} x^{7}}{A^{4} A^{5}} \\
0 & 0 & 0 & 0 & \frac{A^{6}}{A^{5}} & -\frac{x^{5} x^{6}}{A^{5} A^{6}} & -\frac{x^{5} x^{7}}{A^{5} A^{6}} \\
0 & 0 & 0 & 0 & 0 & \frac{x^{7}}{A^{6}} & -\frac{x^{6}}{A^{6}} \\
\frac{x^{1}}{A^{1}} & \frac{x^{2}}{A^{1}} & \frac{x^{3}}{A^{1}} & \frac{x^{4}}{A^{1}} & \frac{x^{5}}{A^{1}} & \frac{x^{6}}{A^{1}} & \frac{x^{7}}{A^{1}}
\end{array}\right] .
$$

Note that the $k$-th row of $A$ is $e_{k}$. Since $d \mathcal{A} \mathcal{A}^{T}$ is skew-symmetric and at the reference point $\mathcal{A}$ is the identity matrix, $d \mathcal{A}$ is skew-symmetric at the reference point. Writing (4.9) in blocks as

$$
\mathcal{A}=\left[\begin{array}{ll}
\tilde{\mathcal{A}} & B \\
C & D
\end{array}\right]
$$

where $\tilde{\mathcal{A}}$ is a $6 \times 6$ matrix, we have that $d \tilde{\mathcal{A}}$ is skew-symmetric and upper triangular, hence $d \tilde{\mathcal{A}}=0$ at the reference point. Let $\imath: S^{6} \hookrightarrow E(7)$ be the inclusion map. Then $\imath^{*}\left(d x^{7}\right)=0$ at the reference point. From (4.8), we have

$$
\left[\begin{array}{c}
d \phi^{1} \\
\vdots \\
d \phi^{6}
\end{array}\right]=\left[\begin{array}{ll}
d \tilde{\mathcal{A}} & d B
\end{array}\right]\left[\begin{array}{c}
d x^{1} \\
\vdots \\
d x^{7}
\end{array}\right] .
$$

Since $d \tilde{\mathcal{A}}=0$ and $\imath^{*}\left(d x^{7}\right)=0$ at the reference point, we have

$$
\imath^{*}\left(d \phi^{j}\right)=0, j=1, \ldots, 6,
$$


at the reference point. Therefore, from (4.6) and (4.10), we have at the reference point

$$
d \theta^{\eta}=-i \sum_{\lambda=1}^{6} d J_{\lambda}^{\eta} \wedge \phi^{\lambda} .
$$

To evaluate the right hand side of (4.11) at the reference point, first we apply $d$ to $(4.3)$ and evaluate at the reference point $(0, \ldots, 0,1)$, to obtain

$$
\left(d J_{\mu}^{\nu}\right)=\left[\begin{array}{cccccc}
0 & -d x^{3} & d x^{2} & -d x^{5} & d x^{4} & -d x^{7} \\
d x^{3} & 0 & -d x^{1} & d x^{6} & -d x^{7} & -d x^{4} \\
-d x^{2} & d x^{1} & 0 & -d x^{7} & -d x^{6} & d x^{5} \\
d x^{5} & -d x^{6} & d x^{7} & 0 & -d x^{1} & d x^{2} \\
-d x^{4} & d x^{7} & d x^{6} & d x^{1} & 0 & -d x^{3} \\
d x^{7} & d x^{4} & -d x^{5} & -d x^{2} & d x^{3} & 0
\end{array}\right]
$$

which implies

$$
\imath^{*}\left(d J_{\lambda}^{\eta}\right)=\left[\begin{array}{cccccc}
0 & -\phi^{3} & \phi^{2} & -\phi^{5} & \phi^{4} & 0 \\
\phi^{3} & 0 & -\phi^{1} & \phi^{6} & 0 & -\phi^{4} \\
-\phi^{2} & \phi^{1} & 0 & 0 & -\phi^{6} & \phi^{5} \\
\phi^{5} & -\phi^{6} & 0 & 0 & -\phi^{1} & \phi^{2} \\
-\phi^{4} & 0 & \phi^{6} & \phi^{1} & 0 & -\phi^{3} \\
0 & \phi^{4} & -\phi^{5} & -\phi^{2} & \phi^{3} & 0
\end{array}\right]
$$

at the reference point. Substituting (4.12) in (4.11) we have

$$
\begin{aligned}
& d \theta^{1}=-2 i \phi^{2} \wedge \phi^{3}-2 i \phi^{4} \wedge \phi^{5}, \\
& d \theta^{2}=2 i \phi^{1} \wedge \phi^{3}+2 i \phi^{4} \wedge \phi^{6}, \\
& d \theta^{3}=-2 i \phi^{1} \wedge \phi^{2}-2 i \phi^{5} \wedge \phi^{6},
\end{aligned}
$$

at the reference point. Since $\theta^{1}=\phi^{1}+i \phi^{6}, \theta^{2}=\phi^{2}+i \phi^{5}$, and $\theta^{3}=\phi^{3}+i \phi^{4}$ at the reference point, by expressing the right hand side of (4.13) in terms of $\theta^{j}, \bar{\theta}^{j}, j=1,2,3$, we have

$$
\left[\begin{array}{l}
d \theta^{1} \\
d \theta^{2} \\
d \theta^{3}
\end{array}\right] \equiv \underbrace{\left[\begin{array}{ccc}
0 & 0 & -i \\
0 & i & 0 \\
-i & 0 & 0
\end{array}\right]}_{\mathcal{T}}\left[\begin{array}{l}
\bar{\theta}^{1} \wedge \bar{\theta}^{2} \\
\bar{\theta}^{1} \wedge \bar{\theta}^{3} \\
\bar{\theta}^{2} \wedge \bar{\theta}^{3}
\end{array}\right] \bmod (\theta)
$$

at $(0, \ldots, 0,1)$, and hence the torsion matrix has full rank. Since the rank of a torsion matrix $\mathcal{T}$ of (4.14) is lower semi-continuous, we conclude that $\left(S^{6}, J\right)$ has type $(1,0)$ and therefore, there are no holomorphic functions other than constants.

\section{References}

[1] H. Ahn and C. K. Han, Local geometry of Levi-forms associated with the existence of complex manifolds and the minimality of generic CR manifolds, J. Geom. Anal., to appear. 
[2] S. Berhanu, P. Cordaro, and J. Hounie, An Introduction to Involutive Structures, Cambridge U. Press, 2008.

[3] R. L. Bryant, S. S. Chern, R. B. Gardner, H. L. Goldschmidt, and P. A. Griffiths, Exterior Differential Systems, Springer-Verlag, New York, 1991.

[4] E. Cartan, Les systemes differentiels exterieurs et leurs applications geometriques, Herman, Paris, 1945

[5] R. Courant and D. Hilbert, Methods of Mathematical Physics, Vol. II, John Wiley and Sons, New York, 1962.

[6] A. Frölicher, Zur Differentialgeometrie der komplexen Strukturen, Math. Ann. 129 (1955), 50-95.

[7] T. Fukami and S. Ishihara, Almost Hermitian structure on $S^{6}$, Tôhoku Math. J. (2) 7 (1955), 151-156.

[8] R. B. Gardner, Invariants of Pfaffian systems, Trans. Amer. Math. Soc. 126 (1967), 514-533.

[9] C. K. Han and K. H. Lee, Integrable submanifolds in almost complex manifolds, J. Geom. Anal. 20 (2010), no. 1, 177-192.

[10] H. Kim and K. H. Lee, Complete prolongation for infinitesimal automorphisms on almost complex manifolds, Math. Z. 264 (2010), no. 4, 913-925.

[11] B. S. Krugilov, Nijenhuis tensors and obstructions to constructing pseudoholomorphic mappings, Mathematical Notes 63 (1998), 476-493.

[12] O. Muškarov, Almost complex manifolds without almost holomorphic functions, C. R. Acad. Bulgare Sci. 34 (1981), no. 9, 1225-1228.

[13] Existence of holomorphic functions on almost complex manifolds, Math. Z. 192 (1986), no. 2, 283-295.

[14] A. Newlander and L. Nirenberg, Complex analytic coordinates in almost complex manifolds, Ann. of Math. (2) 65 (1957), 391-404.

[15] F. Treves, Approximation and representation of functions and distributions annihilated by a system of complex vector fields, École Polytechnique, Centre de Mathematiques, Palaiseau, 1981.

CHONG-KYU HAN

Department of Mathematical Sciences

Seoul National University

SEOUl 151-747, KoreA

E-mail address: ckhan@snu.ac.kr

HYESEON KIM

Department of Mathematical Sciences

Seoul National University

SEOUL 151-747, KoreA

E-mail address: hop222@snu.ac.kr 